of one or both eyes should start with an immediate intravenous injection of dexamethasone $10 \mathrm{mg}$ and then, as in a cardiac disaster, the patient should be rushed to hospital for admission, if possible to an intensive care ward. The cause of the blindness is likely to be an underlying medical condition and it is best to proceed without delay to treat the patient. Dexamethasone should be continued $4 \mathrm{mg}$ intramuscularly every six hours for three days. Prednisolone $20 \mathrm{mg}$ three times daily should be started on the first day and continued as described in your leading article."

I have not, moreoever, recommended temporal artery biopsy in view of the very precarious circulatory balance which may exis in giant cell arteritis between the internal and external carotid circulation. Temporal artery biopsy has disturbed this balance and produced ipsilateral blindness.

\section{GeRald Parsons-Smith}

Redhill, Surrey RH1 4QX

12 Parsons-Smith, B G, in Medical Ophthalmology, ed 1976.

\section{One-visit endoscopic clinic}

SIR,-I was interested to read the paper entitled "Evaluation of one-visit endoscopic clinic for patients with dyspepsia" by Dr A K Beavis and others (26 May, p 1387). This will be a lesson to those who use heavy sedationup to $50 \mathrm{mg}$ of diazepam for such a tolerable procedure.

We undertake on average 20-25 endoscopies a week and most cases are referred directly to the endoscopy unit as day cases. Ninety-five per cent of the patients have no premedication or sedation during the procedure. Full cooperation of the patients is achieved by explanation of the procedure to them by the referring doctor and the endoscopist. It seems that Dr Beavis and his colleagues have achieved such a high success rate (187 out of 200 patients) by having developed a rapport with the patients at the time of their detailed examination. I would agree with them that men and older patients tolerate the procedure well but I find that women, particularly under the age of 30 , are reluctant. It is interesting that $81 \%$ of their patients preferred barium meal examination and only $8 \%$ preferred endoscopy. $\mathrm{Up}$ to two-thirds of our patients prefer endoscopy after realising the value of direct visualisation.

I hope that the barium meal examination in each patient was performed for the purposes of their paper and that it is not a routine practice for each patient to have both endoscopy and a barium meal; otherwise this would invalidate cost-effectiveness and patient convenience.

\section{A N Chatterji}

University Department of
Medicine,
Leicester Royal Infirmary,
Leicester LE2 7LX

\section{Cervical cytology reporting}

SIR,-I should like to comment on the correspondence on this subject by $\operatorname{Dr} R \mathrm{E} \mathrm{G}$ Sloan (19 May, p 1356) and Dr M J Sworn (2 June, $p$ 1489). It has been the concern of many pathologists throughout the country to devise a simple, rapid, and accurate assessment of the cell content of smears for purposes of quality assurance. We in this laboratory have successfully practised such cell scoring for the past 10 years, the screeners entering the relevant data in the research boxes 26-30 at the bottom of the national cytology request/ report form (HMR 101/5) while screening the smear or smears.

The average smear under the $22-50 \mathrm{~mm}$ coverslip, using a $\times 10$ eyepiece and $\times 10$ objective, is approximately 30 fields horizontally and 10 fields vertically, giving a total of around 300 fields. In box 26 the technician scores the total cell conten as $\mathrm{C}_{1}$ if over $10000, \mathrm{C}_{2}$ if over $20000, \mathrm{C}_{3}$ if over 30000 , and $C_{4}$ if over 40000 . In box 27 the number of endocervical cells is similarly scored, together with any metaplastic and transformation zone cells, from $E_{0}$ (no cells) to $E_{4}$, when 10 to 20 sheets of cells exist. Boxes 28 and 29 are used to denote blood (B), pus (P), or spermatozoa (S) in series of degrees according to whether or not their presence obscures a quarter $\left(B_{1}\right)$, half $\left(B_{2}\right)$, threequarters $\left(B_{3}\right)$, or the whole $\left(B_{4}\right)$ of the epithelia element in the smear. This cell scoring is by no means difficult or time-consuming as the technicians become very adept at assessing the average cell content in spite of the widely differing cell content per field. Moreover, this practice keeps the cytotechnicians alert and interested while screenin and provides a greater job satisfaction, especially as they initial the corner of box 22 , where a written report may or may not be recorded. By this method we can rapidly check the detection rate of endocervical cells by one cytotechnician against the laboratory norms. A further check is made by senior or chief technician to note the adequacy of the test and score (when a further initial is placed in the local code box beside box 26), and to assess whether or not the smear needs to be checkscreened. Then that person's initial goes beside that of the screener's in box 22 . The proportion of double-checked reports with two initials amounts on average to $10-15 \%$ of all gvnaecological smears. Such cell scoring helps the pathologist when signing out the report to consider the possible need for a repeat, its time interval, and a suggested further investigation.

I would say that Dr Sworn's rough assessment of $10-15^{\circ}$ of smears lacking endocervical cells is probably a gross underestimate, as many of the published findings quoted have demonstrated that $50 \%$ or more of smears lack these cells-and this is so for those in the child-bearing and sexually active phase of life. ${ }^{12}$ It is obvious that we cannot make the presence or absence of endocervical cells a strict measure of the adequacy of a smear; but such a practice of scoring, when understood by the clinicians and general practitioners served by a laboratory, does help to provide a measure of confidence in a particular test.

\section{O A N Husain}

Division of Pathology, St Stephen's Hospital,
London SW10 9TH

\footnotetext{
${ }^{1}$ Husain, O A N, et al, fournal of Clinical Pathology, ${ }^{2}$ Husain, O A N, Tumori, 1976, 62, 303.
}

SIR,-I was the partner referred to by Dr R E G Sloan (19 May, p 1356). As he stated, we collected reports on all smears over a six month period. Although one technician did request a higher number of repeats than the others, this was far from significant and the numbers involved were not adequate for a firm conclusion to be drawn. As Dr M J Sworn commented (2 June, p 1489), perhaps this technician was the most accurate.

Surely the point of the cervical smear is to exclude malignant or premalignant change, and if it has to be repeated to be certain then this is in the patient's best interest if one accepts the validity of taking the smear in the first instance. Different laboratories obviously have different reporting techniques, and if phrases such as "No malignant cells seen" are used a word with the local pathologist is needed to decide exactly what is meant. The interpretation of technicians and cytologists varies as much as, if not more than, the accuracy of the clinician taking the smear; and it is my impression that the fixing of the smear and the shelf life of the fixative (quoted as indefinite) is more significant than either of the previously mentioned factors.

It seems to be everybody's experience that the smears you are sure are inadequate are reported as normal, and the ones you think are certain to be all right show no endocervical cells.

Cheltenham, Glos GL53 0BA

ROBIN R HARROD

\section{Pressure on tracheal mucosa from cuffed} tubes

SIR,-The use of a low-pressure high-volume cuff (5 May, $p$ 1173) on endotracheal and tracheostomy tubes would appear to offer several theoretical advantages, particularly in the long term $^{12}$; but I have reviewed the experience of our unit using the traditional Portex low-volume, high-pressure cuff in over 1000 consecutive cases of endotracheal intubation and failed to find any cases of tracheal damage or stenosis. My experience of the newer, "floppy" cuff is that the endotracheal tube is difficult to insert via the nose (our usual method for long-term intubation) without bursting the cuff; it sometimes has been difficult to deflate and remove; and the point at which an airtight seal is achieved is as difficult to determine as with the traditional cuff.

It is probably of equal importance to use the correct size of endotracheal tube so that cuff overinflation does not occur, that careful inflation with the minimum volume of air ensures an airtight seal, and that the cuff then remains continuously inflated until it is changed or removed.

Intensive Care Unit,

D W RYAN

Newcastle General Hospital

Newcastle upon Tyne NE4 6BE

1 Cooper, J D, and Grillo, H C, Annals of Surgery, 1969

${ }^{2}$ Crawley, B E, and Cross, D E, Anaesthesia, 1975, 30, 4

\section{Indications for electric convulsion} therapy

SIR,-Inability to cry is a recognised feature of so-called endogenous depression. ECT is said to be more effective in endogenous than in reactive or neurotic depression. I was surprised that Drs David Gill and John Lambourn (5 May, p 1169) did not mention inability to cry as a possible indication for ECT, as in a study I carried out at the University of Sheffield this symptom was a better prognostic sign than early waking, loss of weight, or diurnal variation.

A comparison of the therapeutic effects of unilateral, bifrontal, and bitemporal ECT was carried out. ${ }^{1}$ Subjects completed the Wakefield depression inventory ${ }^{2}$ before and after their course of treatment. The length of course 